www.mdpi.com/journal/applsci

Article

\title{
Ultrasonics Promoted Synthesis of 5-(Pyrazol-4-yl)-4,5-Dihydropyrazoles Derivatives
}

\section{Jorge Trilleras ${ }^{1}$, Efraín Polo ${ }^{1}$, Jairo Quiroga ${ }^{2}$, Justo Cobo ${ }^{3}$ and Manuel Nogueras ${ }^{3, *}$}

1 Research Group of Heterocyclic Compounds, Chemistry Program, Faculty of Basic Sciences, The University of Atlantico, Km 7 Antigua vía Puerto Colombia, Barranquilla-Atlántico, Colombia; E-Mails: jorgetilleras@mail.uniatlantico.edu.co (J.T.); efrain2389@gmail.com (E.P.)

2 Research Group of Heterocyclic Compounds, Department of Chemistry, University of Valle, A. A 25360 Cali, Colombia; E-Mail: jaiquir@univalle.edu.co

3 Department of Inorganic and Organic Chemistry, University of Jaén, 23071 Jaén, Spain; E-Mail: jcobo@ujaen.es

* Author to whom correspondence should be addressed; E-Mail: mmontiel@ujaen.es; Tel.: +34-953-213-087; Fax: +34-618-907-111.

Received: 31 January 2013; in revised form: 26 February 2013 / Accepted: 27 March 2013 / Published: 16 April 2013

\begin{abstract}
A series of new 1,3-diaryl-5-(1-phenyl-3-methyl-5-chloropyrazol-4-yl)-4,5dihydropyrazole derivatives have been synthesized under sonication conditions in ethanol or methanol/glacial acetic acid mixture (5/1 ratio) with two equivalents of hydrazines and seven kinds of chalcone-like heteroanalogues obtained from 5-chloro-3-methyl-1-phenyl$1 H$-pyrazole-4-carbaldehyde. The structures were established on the basis of NMR, IR, MS and element analysis. This method provides several advantages over current reaction methodologies, including a simple work-up procedure, shorter reaction times (2-20 min) and good yields $(65 \%-80 \%)$.
\end{abstract}

Keywords: pyrazolines; cyclocondensation; sonication; chalcones; hydrazines

\section{Introduction}

Pyrazole and pyrazoline (dihydropyrazoles) derivatives are a class of heterocyclic compounds that have drawn much attention, due to their biological and pharmaceutical activities [1]. A brief survey on the biological activities of various pyrazole and pyrazoline derivatives showed anti-inflammatory [2-6], 
antitumor [7-10], antifungal [11-13], antiviral and antibacterial [12,14,15], as well as fluorescent properties [16-21]. In addition to these effects, in the last decade, pyrazolines and substituted pyrazolines have emerged as promising anti-depressant and anti-convulsant agents [22-25]. Of all the synthesized pyrazoline derivatives, the 1,3,5-tri-substituted derivatives are of particular importance. So, it is important to find simple and convenient procedures for pyrazole and pyrazoline preparations with different substituent in their moiety, with the aim of obtaining some novel heterocyclic compounds with potentially enhanced properties.

The development of new, rapid and clean synthetic routes toward focused libraries of nitrogen-containing heterocycles is of great importance to both synthetic and medicinal chemists. They have been reported in literature procedures for the design and development of new heterocycles (pyrazole and pyrazoline derivatives) by means of multistep reactions [26-28], metal-catalyzed synthesis [29,30], domino reaction of 2-acylaziridines with the Huisgen zwitterions [31] and 1,3-dipolar cycloaddition reactions [32] to access important heterobiaryls.

The first synthesis of the pyrazoline framework by the reaction of an $\alpha, \beta$-enone with a hydrazine derivative was published by Fischer and Knoevenagel [33]. Then, the reaction of $\alpha, \beta$-unsaturated aldehydes and ketones with hydrazine derivatives became one of the most popular methods for the synthesis of pyrazolines [34-37].

Cyclization of chalcones, leading to pyridine, pyrimidine and pyrazoline derivatives, has been a developing field within the realm of heterocyclic chemistry for the past several years, because of their ready accessibility and the broad spectrum of biological activity of the products [38-44]. These observations led us to synthesize chalcones and its corresponding pyrazoline, exploring simple procedures.

Sonochemistry is attracting considerable research activity within the synthetic chemistry community, because it offers a new approach to the preparation of organic compounds. In the last two decades, sonochemical methods have become widely used in organic synthesis [45-47]. Nowadays, the ultrasonic irradiation technique has been employed, not only to decrease reaction times, but also to improve yields in a large variety of polyfunctionalized heterocycles. Compared with traditional methods, this method is more convenient and easily controlled. A large number of organic reactions can be carried out in a higher yield shorter reaction time and milder conditions under ultrasound [48-52].

\section{Experimental Section}

\subsection{Apparatus and Analysis}

Melting points were determined using a Thermo Scientific Fluke 51 II, model IA 9100 melting point apparatus and are reported uncorrected. ${ }^{1} \mathrm{H}$ NMR $(400 \mathrm{MHz})$ and ${ }^{13} \mathrm{C}$ NMR $(100 \mathrm{MHz})$ spectra were recorded at room temperature on a Bruker Ultra Shield 400 using tetramethylsilane (TMS) as the internal standard and deuterated chloroform $\left(\mathrm{CDCl}_{3}\right)$ as the solvent. EI-MS were run on a Shimadzu GC-MS 2010 spectrometer, which was operating at $70 \mathrm{eV}$. IR spectra were recorded as $\mathrm{KBr}$ pellets on a Shimadzu FTIR-8400 instrument. The ultrasonic irradiation was performed by using a Branson ultrasonic cleaner bath, model 1510, AC input $115 \mathrm{~V}$, output $50 \mathrm{~W}, 1.9$ liters with a mechanical timer (60 min with continuous hold) and heater switch, $47 \mathrm{KHz}$. High Resolution Mass Spectra (HRMS) 
were recorded in a Waters Micromass AutoSpec NT spectrometer (STIUJA). The elemental analyses have been obtained using a LECO CHNS-900 and Thermo Finnigan FlashEA1112 CHNS-O (STIUJA) elemental analyzers. The hydrazines and solvents used, such as, ethanol, dichloromethane, glacial acetic acid and ethyl acetate, were obtained from Merck Chemical Company. The chalcone-like heteroanalogues 1 were obtained according to the methodology described [39,53,54].

\subsection{General Procedure for the Synthesis of 5-pyrazol-4,5-dihydropyrazoles Derivatives 3}

A solution of equimolar amounts of chalcone-like heteroanalogues 1 (1 mmol) and hydrazine 2 ( $1 \mathrm{mmol})$, using as solvent ethanol or methanol/acetic acid mixture $(5 / 1 \mathrm{ratio}, 10 \mathrm{~mL})$ in an Erlenmeyer, was placed in a water bath and sonicated at ambient conditions $\left(35-40{ }^{\circ} \mathrm{C}\right)$, for an appropriate time (Table 2), until the reaction was completed (the reaction was monitored by TLC). The reaction mixture was then treated with cold ethanol and filtered to leave a solid product, which was crystallized from a hexane/ethanol mixture to yield pure product 3. All the products were characterized by their physical and spectral data (IR, MS, $1 \mathrm{H}$ NMR, ${ }^{13} \mathrm{C} \mathrm{NMR}$ ) and elemental analysis.

\subsubsection{Compound 3a}

5-Chloro-4-(4,5-dihydro-1-phenyl-3-p-tolyl-1H-pyrazol-5-yl)-3-methyl-1-phenyl-1H-pyrazole. Yellow solid, 80\%. mp 133-136 ${ }^{\circ} \mathrm{C} .{ }^{1} \mathrm{H}$ NMR (400 MHz, DMSO- $\left.d_{6}\right) \delta(\mathrm{ppm}): 2.12\left(\mathrm{~s}, 3 \mathrm{H}, \mathrm{CH}_{3}\right), 2.32$ $\left(\mathrm{s}, 3 \mathrm{H}, \mathrm{CH}_{3}\right), 3.32\left(\mathrm{~m}, 1 \mathrm{H}, \mathrm{CH}_{2}\right), 3.92\left(\mathrm{~m}, 1 \mathrm{H}, \mathrm{CH}_{2}\right), 5.66(\mathrm{~m}, 1 \mathrm{H}, \mathrm{CH}), 6.95(\mathrm{t}, 1 \mathrm{H}, \mathrm{Hp}, \mathrm{N}-\mathrm{Ph}$, $J=7.43 \mathrm{~Hz}$ ), 7.17 (d, 2H, Ho, N-Ph, $J=8.28 \mathrm{~Hz}$ ), 7.28 (t, 2H, Hm, N-Ph, $J=8.54 \mathrm{~Hz}$ ), 7.39 (t, 1H, $\mathrm{Hp}, \mathrm{N}-\mathrm{Ph}, J=7.54 \mathrm{~Hz}$ ), 7.47 (t, 2H, Hm, N-Ph, $J=8.28 \mathrm{~Hz}$ ), 7.49 (d, 2H, Hm, 3-aryl, $J=7.54 \mathrm{~Hz}$ ), 7.52 (d, 2H, Ho, N-Ph, $J=8.45 \mathrm{~Hz}), 7.63$ (d, 2H, Ho, 3-aryl, $J=7.53 \mathrm{~Hz}) .{ }^{13} \mathrm{C}$ NMR $\delta(\mathrm{ppm}): 13.3$ $\left(\mathrm{CH}_{3}\right), 14.8\left(\mathrm{CH}_{3}\right), 41.7\left(\mathrm{CH}_{2}\right), 54.3(\mathrm{CH}), 113.8(\mathrm{Cm}, \mathrm{N}-\mathrm{Ph}), 118.5(\mathrm{C} 4$ pyrazole $), 120.1(\mathrm{Cp}, \mathrm{N}-\mathrm{Ph})$, 121.6 (Cp, 3-aryl), 125.0 (Co, N-Ph), 126.8 (Cm, 3-aryl), 127.9 (Cp, N1-Ph), 128.7 (Cm, N1-Ph), 129.1 (Co, N1-Ph), 131.5 (Ci, 3-aryl), 132.0 (Co, 3-aryl), 137.6 (Ci, N1-Ph), 144.3 (C5 pyrazole), 145.8 (C3 pyrazoline), 147.7 (C3 pyrazole). HR-MS Calc. For $\mathrm{C}_{26} \mathrm{H}_{23} \mathrm{ClN}_{4}, 426.1611$, found 426.1618 . FT-IR $\left(\mathrm{KBr}, v\right.$ en $\left.\mathrm{cm}^{-1}\right), 1592(\mathrm{C}=\mathrm{N}, s t), 1502(\mathrm{C}=\mathrm{C}, s t)$. A. E: Calc. For $\mathrm{C}_{26} \mathrm{H}_{23} \mathrm{ClN}_{4} \mathrm{C}: 73.14, \mathrm{H}$ : 5.43, N: 13.12, found C: 73.28, H: 5.93, N: 12.99 .

\subsubsection{Compound 3b}

4-(3-(4-Bromophenyl)-4,5-dihydro-1-phenyl-1H-pyrazol-5-yl)-5-chloro-3-methyl-1-phenyl-1Hpyrazole. Yellow solid, 75\%. mp 163-165 ${ }^{\circ} \mathrm{C} .{ }^{1} \mathrm{H}$ NMR (400 MHz, $\left.\mathrm{CDCl}_{3} \mathrm{RT}\right) \delta$ (ppm): $2.17\left(\mathrm{CH}_{3}\right)$, 3.20-3.79 (m, 2H, CH $), 5.38$ (q, 1H, CH), 6.86 (t, 1H, Hp, N-Ph, J=7.28 Hz), 7.13 (d, 2H, Ho, N-Ph, $J=8.53 \mathrm{~Hz}$ ), 7.25 (t, 2H, Hm, N-Ph, $J=7.28 \mathrm{~Hz}$ ), 7.41 (t, 1H, Hp, N-Ph, $J=7.03 \mathrm{~Hz}$ ), 7.49 (t, 2H, $\mathrm{Hm}, \mathrm{N}-\mathrm{Ph}, J=8.03 \mathrm{~Hz}$ ), 7.54 (d, 2H, Hm, 3-aryl, $J=8.54 \mathrm{~Hz}), 7.55$ (d, 2H, Ho, N-Ph, $J=7.03 \mathrm{~Hz}$ ), $7.63\left(\mathrm{~d}, 2 \mathrm{H}, \mathrm{Ho}, 4-(3\right.$-aryl), $J=8.53 \mathrm{~Hz}) .{ }^{13} \mathrm{C}$ NMR $\delta(\mathrm{ppm}): 13.3\left(\mathrm{CH}_{3}\right), 40.7\left(\mathrm{CH}_{2}\right), 55.3(\mathrm{CH}), 113.4$ (Cm, N-Ph), 117.5 (C4 pyrazole), 119.7 (Cp, N-Ph), 122.6 (Cp, 3-aryl), 124.8 (Co, N-Ph), 127.1 (Cm, 3-aryl), 128.2 (Cp, N1-Ph), 129.0 (Cm, N1-Ph), 129.1 (Co, N1-Ph), 131.5 (Ci, 3-aryl), 131.8 (Co, 3-aryl), 138.0 (Ci, N1-Ph), 144.3 (C5 pyrazole), 145.6 (C3 pyrazoline), 147.7 (C3 pyrazole). MS $(70 \mathrm{eV}) \mathrm{m} / \mathrm{z}(\%)=494 / 492\left(\mathrm{M}^{+2} / \mathrm{M}^{+}, 8 / 29\right), 490(23), 91(100)$, 77(61), 64(28), 51(28). HR-MS Calc. 
For $\mathrm{C}_{25} \mathrm{H}_{20} \mathrm{BrClN}_{4}, 490.0560$, found 490.0579. FT-IR $\left(\mathrm{KBr}, v\right.$ en $\left.\mathrm{cm}^{-1}\right), 1594(\mathrm{C}=\mathrm{N}, s t), 1498(\mathrm{C}=\mathrm{C}$, st). A. E: Calc. For $\mathrm{C}_{25} \mathrm{H}_{20} \mathrm{BrClN}_{4} \mathrm{C}: 61.05, \mathrm{H}: 4.10, \mathrm{~N}: 11.39$, found C: 61.07, H: 3.83, N: 11.28 .

\subsubsection{Compound 3c}

5-Chloro-4-(3-(4-chlorophenyl)-4,5-dihydro-1-phenyl-1H-pyrazol-5-yl)-3-methyl-1-phenyl-1Hpyrazole. Yellow solid, 70\%. mp 153-156 ${ }^{\circ} \mathrm{C} .{ }^{1} \mathrm{H}$ NMR (400 MHz, $\mathrm{CDCl}_{3} \mathrm{RT}$ ) $\delta$ (ppm): 2.07 (s, 3H, $\left.\mathrm{CH}_{3}\right), 3.28\left(\mathrm{~m}, 1 \mathrm{H}, \mathrm{CH}_{2}\right), 3.88\left(\mathrm{~m}, 1 \mathrm{H}, \mathrm{CH}_{2}\right), 5.46(\mathrm{~m}, 1 \mathrm{H}, \mathrm{CH}), 6.77$ (t, 1H, Hp, J= $\left.7.24 \mathrm{~Hz}\right), 7.03$ (d, 2H, Ho, aryl, $J=7.65 \mathrm{~Hz}$ ), 7.21 (t, 2H, Hm, $J=7.24 \mathrm{~Hz}), 7.43-7.54$ (m, 7H, Hm, Hp, Ho aryl, Hp aryl), $7.77(\mathrm{~d}, 2 \mathrm{H}, \mathrm{Ho}, J=8.48 \mathrm{~Hz}) .{ }^{13} \mathrm{C}$ NMR $\delta(\mathrm{ppm}): 12.8\left(\mathrm{CH}_{3}\right), 40.1\left(\mathrm{CH}_{2}\right), 54.4(\mathrm{CH}), 112.8$ (Cm), 117.3 (C4 pyrazole), 119.1 (Cp), 124.4 (Ci), 124.6 (Co), 127.3 (Cm aryl), 128.3 (Cp), 128.7 (Co aryl), $129.0(\mathrm{Cm}), 129.2$ (Co), 131.0 (Ci aryl), 133.1 (Cp aryl), 137.5 (Ci), 143.8 (C5 pyrazole), 146.5 (C3 dihidropyrazole), 147.1 (C3 pyrazole). HR-MS Calc. For $\mathrm{C}_{25} \mathrm{H}_{20} \mathrm{Cl}_{2} \mathrm{~N}_{4} 446.1065$, found 446.1064 . FT-IR $\left(\mathrm{KBr}, v\right.$ en $\left.\mathrm{cm}^{-1}\right), 1598(\mathrm{C}=\mathrm{N}, s t), 1495(\mathrm{C}=\mathrm{C}, s t)$. A. E: Calc. For $\mathrm{C}_{25} \mathrm{H}_{20} \mathrm{Cl}_{2} \mathrm{~N}_{4} \mathrm{C}: 67.12, \mathrm{H}$ : 4.51, N: 12.52, found C: 67.14, H: 4.49, N: 12.51 .

\subsubsection{Compound 3d}

5-Chloro-4-(4,5-dihydro-3-(4-nitrophenyl)-1-phenyl-1H-pyrazol-5-yl)-3-methyl-1-phenyl-1Hpyrazole. Yellow solid, 80\%. mp $178-180{ }^{\circ} \mathrm{C} .{ }^{1} \mathrm{H}$ NMR (400 MHz, $\left.\mathrm{CDCl}_{3} \mathrm{RT}\right) \delta$ (ppm): $2.30\left(\mathrm{CH}_{3}\right)$, $3.17\left(\mathrm{~m}, 1 \mathrm{H}, \mathrm{CH}_{2}\right), 3.44\left(\mathrm{~m}, 1 \mathrm{H}, \mathrm{CH}_{2}\right), 5.11(\mathrm{q}, 1 \mathrm{H}, \mathrm{CH}), 7.48(\mathrm{~m}, 5 \mathrm{H}, \mathrm{CH}), 7.80$ (d, 2H, Hm, 3-aryl, $J=9.1 \mathrm{~Hz}), 8.23(\mathrm{~d}, 2 \mathrm{H}, \mathrm{Ho}, 3$-aryl, $J=9.1 \mathrm{~Hz}) .{ }^{13} \mathrm{C}$ NMR $\delta(\mathrm{ppm}): 13.4\left(\mathrm{CH}_{3}\right), 37.6\left(\mathrm{CH}_{2}\right), 55.3$ $(\mathrm{CH}), 116.3$ (C4, pyrazole), 123.7 (Cm, 3-aryl), $124.6(\mathrm{Co}), 125.9(\mathrm{Cm}), 128.0(\mathrm{Cp}), 128.7$ (Co, 3-aryl), 128.9 (Ci, 3-aryl), 137.7 (Ci), 138.6 (Cp, 3-aryl), 147.1 (C5, pyrazole), 147.8 (C3, pyrazole), 148.5 (C3, pyrazoline). HR-MS Calc. For $\mathrm{C}_{19} \mathrm{H}_{16} \mathrm{ClN}_{5} \mathrm{O}_{2}$ 381.0993, found 381.0983. FT-IR (KBr, $v$ en $\left.\mathrm{cm}^{-1}\right), 1595(\mathrm{C}=\mathrm{N}, s t), 1502(\mathrm{C}=\mathrm{C}, s t)$. A. E: Calc. For $\mathrm{C}_{19} \mathrm{H}_{16} \mathrm{ClN}_{5} \mathrm{O}_{2} \mathrm{C}: 59.77, \mathrm{H}: 4.22, \mathrm{~N}: 18.34$, found $\mathrm{C}: 59.28, \mathrm{H}: 3.93, \mathrm{~N}: 17.99$.

\subsubsection{Compound $\mathbf{3 e}$}

5-Chloro-4-(4,5-dihydro-3-(4-methoxyphenyl)-1-phenyl-1H-pyrazol-5-yl)-3-methyl-1-phenyl-1H-

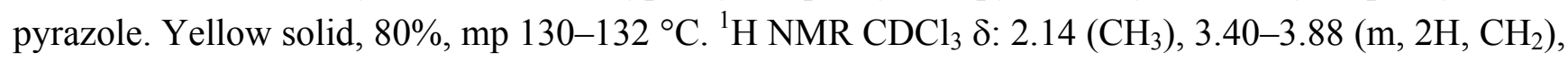
$3.91\left(\mathrm{~s}, 3 \mathrm{H}, \mathrm{OCH}_{3}\right), 5.25$ (q, 1H, CH), 6.43 (d, 2H, Hm 4-(3-aryl), $\left.J=8.78 \mathrm{~Hz}\right), 7.68$ (d, 2H, Ho 4-(3-aryl), $J=8.79 \mathrm{~Hz}), 7.12$ (d, 2H, Ho 4-( $N$-aryl) $J=9.07 \mathrm{~Hz}), 7.15$ (d, 2H, Hm 4-( $N$-aryl), $J=9.04 \mathrm{~Hz}$ ), 7.39 (t, 1H, Hp, N-Ph), 7.47 (t, 2H, Hm, N-Ph), 7.52 (d, 2H, Ho, N-Ph, J = 7.53 Hz), 7.03 (d, 2H, CHo, N1-aryl, $J=8.78 \mathrm{~Hz}$ ), 7.19 (d, 2H, CHm, N1-aryl, $J=9.04 \mathrm{~Hz}), 7.40$ (m, 3H, CHp N-Ph, CHm C3-aryl, $J=8.53 \mathrm{~Hz}$ ), 7.49 (t, 2H, CHm N-Ph), 7.54 (d, 2H, CHo-Ph), 7.68 (d, 2H, CHo C3-aryl, $J=8.54 \mathrm{~Hz}) .{ }^{13} \mathrm{C}$ NMR $\delta(\mathrm{ppm}): 12.7\left(\mathrm{CH}_{3}\right), 41.0\left(\mathrm{CH}_{2}\right), 55.1(\mathrm{CH}), 114.0(\mathrm{C} 4$, pyrazole $), 123.8(\mathrm{Cm}$, 3-aryl), 124.6 (Co), 126.9 (Cm), 128.1 (Cp), 128.8 (Co, 3-aryl), 129.4 (Ci, 3-aryl), 137.8 (Ci), 140.0 (Cp, 3-aryl), 147.7 (C5, pyrazole), 148.9 (C3, pyrazole), 151.7 (C3, pyrazoline). MS (70 eV) $m / z(\%)=442\left(\mathrm{M}^{+2}, 96\right), 440(100), 405(59), 91(75), 77(83)$. FT-IR $\left(\mathrm{KBr}, v\right.$ en cm $\left.{ }^{-1}\right), 1597(\mathrm{C}=\mathrm{N}$, $s t), 1497(\mathrm{C}=\mathrm{C}, s t)$. A. E: Calc. For $\mathrm{C}_{26} \mathrm{H}_{23} \mathrm{ClN}_{4} \mathrm{O}$ C: $70.50, \mathrm{H}: 5.23, \mathrm{~N}: 12.65$, found C: 70.08, H: 5.03, $\mathrm{N}: 11.99$. 


\subsubsection{Compound $\mathbf{3 f}$}

5-Chloro-4-(4,5-dihydro-3-(3,4,5-trimethoxyphenyl)-1-phenyl-1H-pyrazol-5-yl)-3-methyl-1-phenyl

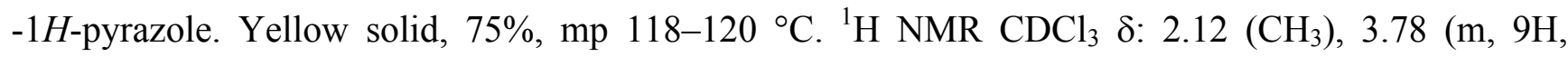
methoxyl, 1H, $\left.\mathrm{CH}_{2}\right), 3.42-3.48,3.90-3.95\left(\mathrm{~m}, 2 \mathrm{H}, \mathrm{CH}_{2}\right), 5.34-5.39$ (q, 1H, CH), $6.88(\mathrm{t}, 1 \mathrm{H}, \mathrm{Hp}$, N-Ph), 7.19 (d, 2H, Ho, N-Ph), 7.25 (t, 2H, Hm, N-Ph), 7.38 (t, 1H, Hp, N1-Ph), 7.49 (t, 2H, Hm, N1-Ph), 7.51 (d, 2H, Hm, 4-(3-aryl), $J=8.53 \mathrm{~Hz}$ ), 7.55 (d, 2H, Ho, N1-Ph), 7.60 (d, 2H, Ho, 4-(3-aryl), $J=8.55 \mathrm{~Hz})$. FT-IR $\left(\mathrm{KBr}, v \mathrm{en} \mathrm{cm}^{-1}\right), 1595(\mathrm{C}=\mathrm{N}, s t), 1500(\mathrm{C}=\mathrm{C}, s t)$.

\subsubsection{Compound $3 g$}

4-(3-(Benzo[d][1,3]dioxol-6-yl)-4,5-dihydro-1-phenyl-1H-pyrazol-5-yl)-5-chloro-3-methyl-1phenyl-1H-pyrazole. Brown solid, 65\%, mp 220-222 ${ }^{\circ} \mathrm{C} .{ }^{1} \mathrm{H} \mathrm{NMR} \mathrm{CDCl}_{3}$ \%: $2.79\left(\mathrm{CH}_{3}\right), 3.52-3.57$, 3.80-3.88 (m, 2H, $\mathrm{CH}_{2}$ ), 5.90 (s, 2H, $\mathrm{CH}_{2}$-dioxol), 5.38-5.41(q, 1H, CH), 6.88 (t, 1H, Hp, N-Ph), 7.19 (d, 2H, Ho, N-Ph), 7.25 (t, 2H, Hm, N-Ph), 7.38 (t, 1H, Hp, N1-Ph), 7.49 (t, 2H, Hm, N1-Ph), 7.51 (d, 2H, Hm, 4-(3-aryl), $J=8.53 \mathrm{~Hz}), 7.55$ (d, 2H, Ho, N1-Ph), 7.60 (d, 2H, Ho, 4-(3-aryl), $J=8.55 \mathrm{~Hz}$ ). ${ }^{13} \mathrm{C}$ NMR $\delta(\mathrm{ppm}): 12.9\left(\mathrm{CH}_{3}\right), 41.2\left(\mathrm{CH}_{2}\right), 55.3(\mathrm{CH}), 114.2$ (Co, 3-aryl), $119.1(\mathrm{C} 4$, pyrazole), 124.8 (Co), $127.1(\mathrm{Cm}), 128.4(\mathrm{Cp}), 133.8$ (Ci), 149.1 (C5, pyrazole). HR-MS Calc. For $\mathrm{C}_{26} \mathrm{H}_{21} \mathrm{ClN}_{4} \mathrm{O}_{2}$ 456.2112, found 456.1217. FT-IR (KBr, v en cm-1), $1598(\mathrm{C}=\mathrm{N}, s t), 1498(\mathrm{C}=\mathrm{C}, s t)$.

\subsubsection{Compound $\mathbf{3 h}$}

5-Chloro-4-(1-(4-chlorophenyl)-4,5-dihydro-3-p-tolyl-1H-pyrazol-5-yl)-3-methyl-1-phenyl$1 H$-pyrazole. Yellow solid, 80\%, mp $158-160{ }^{\circ} \mathrm{C} .{ }^{1} \mathrm{H}$ NMR $\left(400 \mathrm{MHz}, \mathrm{CDCl}_{3} \mathrm{RT}\right) \delta$ (ppm): 2.13 $\left(\mathrm{CH}_{3}\right), 2.38\left(\mathrm{CH}_{3}\right), 3.20\left(\mathrm{~m}, 1 \mathrm{H}, \mathrm{CH}_{2}\right), 3.79\left(\mathrm{~m}, 2 \mathrm{H}, \mathrm{CH}_{2}\right), 5.29$ (q, 1H, $\left.\mathrm{CH}\right), 7.01$ (d, 2H, Hm, $N$-aryl, $J=9.11 \mathrm{~Hz}), 7.16$ (d, 2H, Ho, $N$-aryl, $J=9.09 \mathrm{~Hz}), 7.21$ (d, 2H, Hm, 3-aryl, $J=7.86 \mathrm{~Hz}), 7.39$ (t, 1H, $\mathrm{Hp}, J=7.24 \mathrm{~Hz}), 7.47$ (t, 2H, Hm, $J=7.86 \mathrm{~Hz}$ ), 7.52 (d, 2H, Ho, $J=7.24 \mathrm{~Hz}$ ), 7.63 (d, 2H, Ho, 3-aryl, $J=8.27 \mathrm{~Hz}) .{ }^{13} \mathrm{C}$ NMR $\delta(\mathrm{ppm}): 13.3\left(\mathrm{CH}_{3}\right), 21.4\left(\mathrm{CH}_{3}\right), 41.2\left(\mathrm{CH}_{2}\right), 55.1(\mathrm{CH}), 114.3(\mathrm{Co}, N$-aryl), 117.3 (C4, pyrazole), 124.0 (Ci, N-aryl), 124.8 (Co), 125.7 (Co, 3-aryl), 128.2 (Cp), 128.9 (Cm, $\mathrm{N}$-aril), 129.0 (Cm), 129.4 (Cm, 3-aryl), 133.9 (Ci, 3-aryl), 138.0 (Ci), 139.1 (Cp, 3-aryl), 143.2 (Cp, N-aryl), 147.6 (C3, pyrazole), 147.8 (C3, pyrazoline), 149.0 (C5, pyrazole). HR-MS Calc. For $\mathrm{C}_{26} \mathrm{H}_{22} \mathrm{Cl}_{2} \mathrm{~N}_{4} 460.1222$, found 460.1217. FT-IR $\left(\mathrm{KBr}, v\right.$ en $\left.\mathrm{cm}^{-1}\right), 1592(\mathrm{C}=\mathrm{N}, s t), 1491(\mathrm{C}=\mathrm{C}, s t)$.

\subsubsection{Compound $\mathbf{3 i}$}

4-(1,3-bis(4-Chlorophenyl)-4,5-dihydro-1H-pyrazol-5-yl)-5-chloro-3-methyl-1-phenyl-1H-pyrazole. Yellow solid, 70\%. mp 150-152 ${ }^{\circ} \mathrm{C} .{ }^{1} \mathrm{H}$ NMR (400 MHz, $\left.\mathrm{CDCl}_{3} \mathrm{RT}\right) \delta(\mathrm{ppm}): 2.14\left(\mathrm{CH}_{3}\right), 3.21$ (m, $\left.1 \mathrm{H}, \mathrm{CH}_{2}\right), 3.80\left(\mathrm{~m}, 1 \mathrm{H}, \mathrm{CH}_{2}\right), 5.36(\mathrm{q}, 1 \mathrm{H}, \mathrm{CH}), 7.03$ (d, 2H, Ho, N1-aryl, J = 8.78 Hz), 7.19 (d, 2H, Hm, N1-aryl, $J=9.04 \mathrm{~Hz}$ ), 7.40 (m, 3H, Hp N-Ph, Hm C3-aryl, $J=8.53 \mathrm{~Hz}$ ), 7.49 (t, 2H, Hm N-Ph), 7.54 (d, 2H, Ho Ph, $J=8.28 \mathrm{~Hz}$ ), 7.68 (d, 2H, Ho C3-aryl, $J=8.54 \mathrm{~Hz}$ ). ${ }^{13} \mathrm{C}$ NMR $\delta$ (ppm): 12.9 $\left(\mathrm{CH}_{3}\right), 40.6\left(\mathrm{CH}_{2}\right), 54.9(\mathrm{CH}), 114.1(\mathrm{Cm}, \mathrm{N} 1$-aryl), 116.8 (C4 pyrazole), 124.2 (Ci N1-aryl), 124.5 (Co N1-aryl), 124.7 (C5 pyrazole), 126.6 (Cm C3-aryl), 127.9 (Cp Ph), 128.6 (Co C3-aryl), 128.7 (Co Ph), 130.5 (Ci C3-aryl), 134.4 (Cp C3-aryl), 137.6 (Ci-Ph), 142.5 (Ci N1-aryl), 145.9 (C3), 147.3 (C3 pyrazole). MS $(70 \mathrm{eV}) m / z(\%)=485 / 483\left(\mathrm{M}^{+5} / \mathrm{M}^{+3}, 3 / 9\right), 484 / 482\left(\mathrm{M}^{+4} / \mathrm{M}^{+2}, 11 / 31\right), 480\left(\mathrm{M}^{+}, 33\right)$, 
321/320/319 (7/4/16), 139/137 (4/11), 127/125 (26/73), 113/111 (5/15), 99/97 (5/20), 87/85 (3/12), 83/81 (23/52), 79/77 (6/20), 71/69 (21/100), 57 (33), 55 (29). HR-MS Calc. For $\mathrm{C}_{25} \mathrm{H}_{19} \mathrm{Cl}_{3} \mathrm{~N}_{4}$ 480.0675, found 480.0663. FT-IR $\left(\mathrm{KBr}, v \mathrm{en} \mathrm{cm}^{-1}\right), 1584(\mathrm{C}=\mathrm{N}, s t), 1488(\mathrm{C}=\mathrm{C}, s t)$.

\subsubsection{Compound $\mathbf{3 j}$}

5-Chloro-4-(1-(4-chlorophenyl)-4,5-dihydro-3-(4-methoxyphenyl)-1H-pyrazol-5-yl)-3-methyl1-phenyl-1H-pyrazole. Yellow solid, 80\%. mp $128-130{ }^{\circ} \mathrm{C} .{ }^{1} \mathrm{H}$ NMR (400 $\left.\mathrm{MHz} \mathrm{CDCl}_{3} \mathrm{RT}\right) \delta(\mathrm{ppm})$ : $2.13\left(\mathrm{CH}_{3}\right), 3.19\left(\mathrm{~m}, 1 \mathrm{H}, \mathrm{CH}_{2}\right), 3.78\left(\mathrm{~m}, 1 \mathrm{H}, \mathrm{CH}_{2}\right), 3.85\left(\mathrm{~s}, 3 \mathrm{H}, \mathrm{OCH}_{3}\right), 5.27(\mathrm{q}, 1 \mathrm{H}, \mathrm{CH}), 6.93(\mathrm{~d}, 2 \mathrm{H}$, Hm 3-aryl, $J=8.78 \mathrm{~Hz}$ ), 7.00 (d, 2H, Ho $N$-aryl $J=9.03 \mathrm{~Hz}$ ), 7.15 (d, 2H, Hm $N$-aryl, $J=9.04 \mathrm{~Hz}$ ), 7.39 (t, 1H, Hp, N-Ph, $J=7.78$ Hz), 7.47 (t, 2H, Hm, N-Ph, J=8.03 Hz), 7.52 (d, 2H, Ho, N-Ph, $J=7.53 \mathrm{~Hz}), 7.68$ (d, 2H, Ho 3-aryl, $J=8.79 \mathrm{~Hz}) .{ }^{13} \mathrm{C}$ NMR $\delta(\mathrm{ppm}): 13.3\left(\mathrm{CH}_{3}\right), 41.6\left(\mathrm{CH}_{2}\right), 55.4$ $\left(\mathrm{OCH}_{3}\right), 55.7(\mathrm{CH}), 114.8(\mathrm{Cm}, 3$-aryl), 128.0 (Co, 3-aryl), 125.7 (Ci, 3-aryl), 161.3 (Cp, 3-aryl), 148.6 (C3 pyrazoline), 118.1 (C4 pyrazole), 124.0 (Co, N1-aryl), 124.6 (Ci, N1-aryl), 114.9 (Cm, N1-aryl), 144.2 (C5 pyrazole), 148.3 (C3 pyrazole), 137.8 (Ci, N-Ph), 125.6 (Co, N-Ph), 128.9 (Cp, N-Ph), $129.6(\mathrm{Cm}, \mathrm{N}-\mathrm{Ph})$. MS (70 eV) $m / z(\%)=480 / 478\left(\mathrm{M}^{+2} / \mathrm{M}^{+}, 11 / 71\right), 477 / 475(30 / 100), 315$ (46), 127 (23), 125 (64), 90 (28), 77 (56), 51 (30). FT-IR (KBr, v en cm $\left.{ }^{-1}\right), 1597$ (C=N, st), 1498 (C=C, st).

\section{Results and Discussion}

We continue our study to obtain functionalized heterocycles through the development of synthetic strategies. The starting compounds $\mathbf{1}$ were synthesized by Claisen-Schmidt condensation of 5-chloro-3-methyl-1-phenyl-1H-pyrazole-4-carbaldehyde with acetophenones [53,54]. As part of our ongoing research on the application of ultrasonic irradiation as a clean and useful technique in organic synthesis, we described in this work the synthesis of 5-(pyrazol-4-yl)-4,5-dihydropyrazole derivatives under ultrasound irradiation (Scheme 1).

Scheme 1. Synthesis of 5-(pyrazol-4-yl)-4,5-dihydropyrazole derivatives.

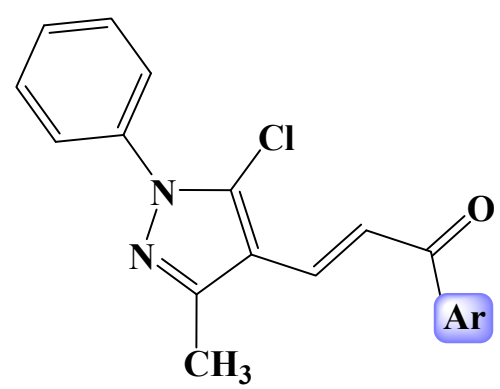

1

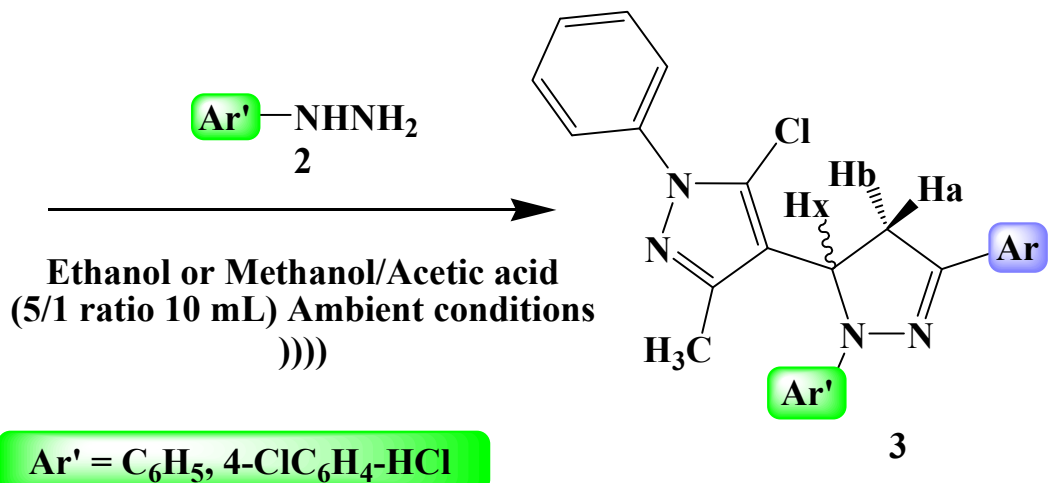

$A r^{\prime}=\mathrm{C}_{6} \mathrm{H}_{5}, 4-\mathrm{ClC}_{6} \mathrm{H}_{4}-\mathrm{HCl}$

We preliminarily examined the cyclocondensation reaction of (E)-3-(5-chloro-3-methyl-1-phenyl1H-pyrazol-4-yl)-1-arylprop-2-en-1-one 1 with hydrazines in the presence of ethanol or methanol and acetic acid as the catalyst under sonication. To achieve suitable reaction conditions in terms of reaction time and catalysis at ambient conditions, we tested different proportions of a mixture of ethanol/methanol and acetic acid. The results are summarized in Table 1. 
Table 1. Effect of reaction conditions. Green factors.

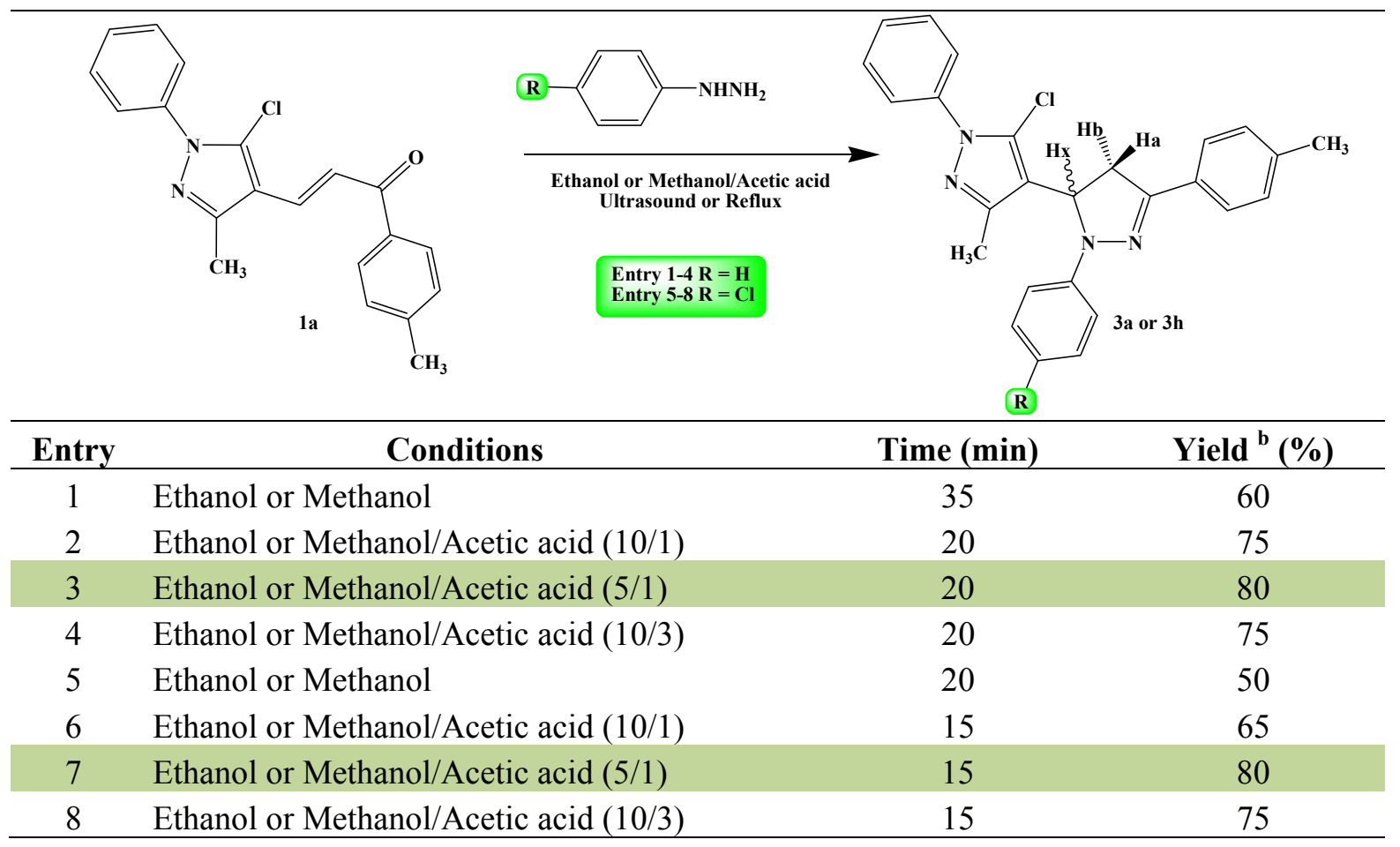

${ }^{\mathrm{b}}$ Isolated yields using ethanol as solvent.

The reaction worked out best under sonication conditions in a mixture of ethanol or methanol/acetic acid $(5 / 1)$ at ambient temperature $\left(35-40{ }^{\circ} \mathrm{C}\right)$ to provide good yield $(65 \%-80 \%)$ in a short time (2-20 $\mathrm{min}$ ), and the results are summarized in Table 2. To develop the scope of the reaction, we were encouraged to extend this reaction to a variety of $(E)$-3-(5-chloro-3-methyl-1-phenyl-1H-pyrazol-4-yl)1-arylprop-2-en-1-one 1 with different substituents under the determined optimum conditions.

Table 2. The synthesized 1,3-diaryl-5-(1-phenyl-3-methyl-5-chloro-pyrazol)-4,5dihydropyrazole derivatives under ultrasonic irradiation at ambient conditions $\left(35-40{ }^{\circ} \mathrm{C}\right)$.

\begin{tabular}{cccccc}
\hline Compound 3 & $\mathbf{A r}$ & $\mathbf{A r}$ & Time reaction (min) & M.p. ${ }^{\circ} \mathbf{C}$ & Yield (\%) \\
\hline $\mathrm{a}$ & $4-\mathrm{H}_{3} \mathrm{CC}_{6} \mathrm{H}_{4}$ & $\mathrm{C}_{6} \mathrm{H}_{5}$ & 20 & $133-135$ & 80 \\
$\mathrm{~b}$ & $4-\mathrm{BrC}_{6} \mathrm{H}_{4}$ & $\mathrm{C}_{6} \mathrm{H}_{5}$ & 10 & $163-165$ & 75 \\
$\mathrm{c}$ & $4-\mathrm{ClC}_{6} \mathrm{H}_{4}$ & $\mathrm{C}_{6} \mathrm{H}_{5}$ & 15 & $153-155$ & 70 \\
$\mathrm{~d}$ & $4-\mathrm{O}_{2} \mathrm{NCC}_{6} \mathrm{H}_{4}$ & $\mathrm{C}_{6} \mathrm{H}_{5}$ & 20 & $178-180$ & 80 \\
$\mathrm{e}$ & $4-\mathrm{H}_{3} \mathrm{COC}_{6} \mathrm{H}_{4}$ & $\mathrm{C}_{6} \mathrm{H}_{5}$ & 3 & $130-132$ & 80 \\
$\mathrm{f}$ & $3,4,5-t r i-\mathrm{H}_{3} \mathrm{COC}_{6} \mathrm{H}_{2}$ & $\mathrm{C}_{6} \mathrm{H}_{5}$ & 2 & $118-120$ & 75 \\
$\mathrm{~g}$ & $3,4-\mathrm{OCH}_{2} \mathrm{OC}_{6} \mathrm{H}_{3}$ & $\mathrm{C}_{6} \mathrm{H}_{5}$ & 5 & $220-222$ & 65 \\
$\mathrm{~h}$ & $4-\mathrm{H}_{3} \mathrm{CC}_{6} \mathrm{H}_{4}$ & $4-\mathrm{ClC}_{6} \mathrm{H}_{4}$ & 15 & $158-160$ & 80 \\
$\mathrm{i}$ & $4-\mathrm{ClC}_{6} \mathrm{H}_{4}$ & $4-\mathrm{ClC}_{6} \mathrm{H}_{4}$ & 10 & $150-152$ & 70 \\
$\mathrm{j}$ & $4-\mathrm{H}_{3} \mathrm{COC}_{6} \mathrm{H}_{4}$ & $4-\mathrm{ClC}_{6} \mathrm{H}_{4}$ & 10 & $128-130$ & 80 \\
\hline
\end{tabular}

We found that the results were excellent compared with 5-pyrazole-4,5-dihidropyrazoline derivatives reported in the literature [55]. Thus, ultrasonic irradiation was found to have a beneficial effect on the synthesis of 1,3-diaryl-5-(1-phenyl-3-methyl-5-chloro-pyrazol)-4,5-dihydropyrazole derivatives, which was superior to the traditional method with respect to yields, reaction times, 
simplicity and safety. The impact of acoustic energy was evident in reduction of the processing time; a physical process that builds, enlarges and collapses gaseous and vaporous cavities in an irradiated liquid, hence enhancing the mass transfer and allowing chemical reactions to occur [56-58].

To the best of our knowledge, this new procedure provides the first example of an efficient and ultrasound-promoted approach for the synthesis of 1,3-diaryl-5-(1-phenyl-3-methyl-5-chloro-pyrazol)4,5-dihydropyrazoles. This method is the most simple and convenient and would be applicable for the synthesis of different types of nitrogen-containing heterocyclic compounds. The structures of all the synthesized compounds were established by their NMR, IR, MS and analysis elemental.

The FT-IR spectra of synthesized 5-pyrazol-4,5-dihydropyrazole derivatives $\mathbf{3}$ showed bands at stretching frequencies in the range of $1584-1598 \mathrm{~cm}^{-1}$ and $1488-1502 \mathrm{~cm}^{-1}$, which are characteristic of $-\mathrm{C}=\mathrm{N}$ and $-\mathrm{C}=\mathrm{C}$ groups. No peak appeared in the range of $1650-1750 \mathrm{~cm}^{-1}$, which indicated the disappearance of the carbonyl group $(\mathrm{C}=\mathrm{O})$ of the $(E)-3$-(5-chloro-3-methyl-1-phenyl-1H-pyrazol-4yl)-1-arylprop-2-en-1-one 1 . The ${ }^{1} \mathrm{H}$ NMR spectrum for compound 3 showed proton signals of the pyrazoline moiety as an ABX-type spin system, and the proton signals were observed as double doublets, due to the spin coupling in the range of 3.17-3.95 ppm. The signal of $-\mathrm{CH}_{3}$ pyrazole and aryl protons in compound was observed between 2.07-2.79 and 6.43-8.23 ppm, respectively.

\section{Conclusions}

The ultrasound promoted reaction of (E)-3-(5-chloro-3-methyl-1-phenyl-1H-pyrazol-4-yl)-1arylprop-2-en-1-one with hydrazines afforded the corresponding 1,3-diaryl-5-(1-phenyl-3-methyl-5chloro-pyrazol)-4,5-dihydropyrazole derivatives, good yields and short reaction times at ambient conditions in a simple, facile and efficient fashion. Due to the broad spectrum of biological activities of pyrazolines, evaluation of the biological activity and fluorescence properties of the new compounds are in progress.

\section{Acknowledgments}

The authors thank "Servicios Técnicos de Investigación of Universidad de Jaén" and the staff for data collection and the Consejería de Innovación, Ciencia y Empresa (Junta de Andalucía, Spain) at the Universidad de Jaén for financial support. JT and JQ thank COLCIENCIAS, UNIATLANTICO (Universidad del Atlántico, Colombia) and UNIVALLE (Universidad del Valle, Colombia) for financial support.

\section{Conflict of Interest}

The authors declare no conflict of interest.

\section{References}

1. Yusuf, M.; Jain, P. Synthetic and biological studies of pyrazolines and related heterocyclic compounds. Arab. J. Chem. 2011, doi:10.1016/j.arabjc.2011.09.013. 
2. Bano, S.; Javed, K.; Ahmad, S.; Rathish, I.G.; Singh, S.; Alam, M.S. Synthesis and biological evaluation of some new 2-pyrazolines bearing benzene sulfonamide moiety as potential anti-inflammatory and anti-cancer agents. Eur. J. Med. Chem. 2011, 46, 5763-5768.

3. Khode, S.; Maddi, V.; Aragade, P.; Palkar, M.; Ronad, P.K.; Mamledesai, S.; Thippeswamy, A.H.M.; Satyanarayana, D. Synthesis and pharmacological evaluation of a novel series of 5-(substituted)aryl-3-(3-coumarinyl)-1-phenyl-2-pyrazolines as novel anti-inflammatory and analgesic agents. Eur. J. Med. Chem. 2009, 44, 1682-1688.

4. Rathish, I.G.; Javed, K.; Ahmad, S.; Bano, S.; Alam, M.S.; Pillai, K.K.; Singh, S.; Bagchi, V. Synthesis and antiinflammatory activity of some new 1,3,5-trisubstituted pyrazolines bearing benzene sulfonamide. Bioorg. Med. Chem. Lett. 2009, 19, 255-258.

5. Schmidt, A.; Dreger, A. Recent advances in the chemistry of pyrazoles. Properties, biological activities, and syntheses. Curr. Org. Chem. 2011, 15, 1423-1463.

6. Abd-El Gawad, N.M.; Hassan, G.S.; Georgey, H.H. Design and synthesis of some pyrazole derivatives of expected anti-inflammatory and analgesic activities. Med. Chem. Res. 2012, 21, 983-994.

7. Insuasty, B.; Chamizo, L.; Muñoz, J.; Tigreros, A.; Quiroga, J.; Abonía, R.; Nogueras, M.; Cobo, J. Synthesis of 1-substituted 3-aryl-5-aryl(hetaryl)-2-pyrazolines and study of their antitumor activity. Arch. Pharm. 2012, 345, 275-286.

8. Al-Saadi, M.S.; Rostom, S.A.F.; Faidallah, H.M. 3-Methyl-2-(4-substitutedphenyl)-4,5dihydronaphtho[1,2-c]-pyrazoles: Synthesis and in vitro biological evaluation as antitumour agents. Arch. Pharm. 2008, 341, 181-190.

9. Insuasty, B.; García, A.; Quiroga, J.; Abonía, R.; Ortiz, A.; Nogueras, M.; Cobo, J. Efficient microwave-assisted synthesis and antitumor activity of novel 4,4'-methylenebis[2-(3-aryl-4,5dihydro-1H-pyrazol-5-yl)phenols]. Eur. J. Med. Chem. 2011, 46, 2436-2440.

10. Insuasty, B.; Tigreros, A.; Orozco, F.; Quiroga, J.; Abonía, R.; Nogueras, M.; Sánchez, A.; Cobo, J. Synthesis of novel pyrazolic analogues of chalcones and their 3-aryl-4-(3-aryl-4,5dihydro-1H-pyrazol-5-yl)-1-phenyl-1H-pyrazole derivatives as potential antitumor agents. Bioorg. Med. Chem. 2010, 18, 4965-4974.

11. Deng, H.; Yu, Z.Y.; Shi, G.Y.; Chen, M.J.; Tao, K.; Hou, T.P. Synthesis and in vitro antifungal evaluation of 1,3,5-trisubstituted-2-pyrazoline derivatives. Chem. Biol. Drug Des. 2012, 79, 279-289.

12. Parashar, B.; Jain, A.; Bharadwaj, S.; Sharma, V.K. Synthesis and pharmacological properties of some novel pyrazolidine and pyrazole derivatives. Med. Chem. Res. 2012, 21, 1692-1699.

13. Gupta, M.; Paul, S.; Gupta, R. Efficient and novel one-pot synthesis of antifungal active 1-substituted-8-aryl-3-alkyl/aryl-4H-pyrazolo[4,5-f][1,2,4]triazolo[4,3- $b][1,2,4]$ triazepines using solid support. Eur. J. Med. Chem. 2011, 46, 631-635.

14. Keter, F.K.; Darkwa, J. Perspective: The potential of pyrazole-based compounds in medicine. Biometals 2012, 25, 9-21.

15. Agrawal, M.; Sonar, P.K.; Saraf, S.K. Synthesis of 1,3,5-trisubstituted pyrazoline nucleus containing compounds and screening for antimicrobial activity. Med. Chem. Res. 2012, 21, $3376-3381$. 
16. Gong, Z.-L.; Xie, Y.-S.; Zhao, B.-X.; Lv, H.-S.; Liu, W.-Y. The synthesis, X-ray crystal structure and optical properties of novel 5-aryl-3-ferrocenyl-1-pyridazinyl-pyrazoline derivatives. J. Fluoresc. 2011, 21, 355-364.

17. Gong, Z.-L.; Zhao, B.-X.; Liu, W.-Y.; Lv, H.-S. A new highly selective "turn on" fluorescent sensor for zinc ion based on a pyrazoline derivative. J. Photochem. Photobiol. A 2011, 218, 6-10.

18. Liu, W.-Y.; Xie, Y.-S.; Zhao, B.-X.; Wang, B.-S.; Lv, H.-S.; Gong, Z.-L.; Song, L.; Zheng, L.-W. The synthesis, X-ray crystal structure and optical properties of novel 5-aryl-1-arylthiazolyl-3ferrocenyl-pyrazoline derivatives. J. Photochem. Photobiol. A 2010, 214, 135-144.

19. Bian, B.S.; Ji, S.; Shi, H. Synthesis and fluorescent property of some novel bischromophore compounds containing pyrazoline and naphthalimide groups. Dye Pigment. 2008, 76, 348-352.

20. Tao, Y.T.; Balasubramaniam, E. Organic light-emitting diodes based on variously substituted pyrazoloquinolines as emitting material. J. Matr. Chem. 2001, 13, 1207-1212.

21. Wang, P.; Onozawa-Komatsuzaki, N.; Himeda, Y.; Sugihara, H.; Arakawa, H.; Kasuga, K. 3-(2-Pyridyl)-2-pyrazoline derivatives: Novel fluorescent probes for $\mathrm{Zn}^{2+}$ ion. Tetrahedron Lett. 2001, 42, 9199-9201.

22. Gok, S.; Demet, M.M.; Özdemir, A.; Turan-Zitouni, G. Evaluation of antidepressant-like effect of 2-pyrazoline derivatives. Med. Chem. Res. 2010, 19, 94-101.

23. Kaplancikli, Z.A.; Özdemir, A.; Turan-Zitouni, G.; Altintop, M.D.; Can, O.D. New pyrazoline derivatives and their antidepressant activity. Eur. J. Med. Chem. 2010, 45, 4383-4387.

24. Amnerkar, N.D.; Bhusari, K.P. Synthesis, anticonvulsant activity and 3D-QSAR study of some prop-2-eneamido and 1-acetyl-pyrazolin derivatives of aminobenzothiazole. Eur. J. Med. Chem. 2010, 45, 149-159.

25. Siddiqui, N.; Alam, P.; Ahsan, W. Design, synthesis, and in-vivo pharmacological screening of N,3-(substituteddiphenyl)-5-phenyl-1H-pyrazoline-1-carbothioamide derivatives. Arch. Pharm. 2009, 342, 173-181.

26. De Los Santos, J.M.; López, Y.; Aparicio, D.; Palacios, F. A convenient synthesis of substituted pyrazolidines and azaproline derivatives through highly regio- and diastereoselective reduction of 2-pyrazolines. J. Org. Chem. 2008, 73, 550-557.

27. Jung, M.E.; Min, S.-J.; Houk, K.N.; Ess, D. Synthesis and relative stability of 3,5-diacyl-4,5dihydro- $1 H$-pyrazoles prepared by dipolar cycloaddition of enones and R-diazoketones. J. Org. Chem. 2004, 69, 9085-9089.

28. Suga, H.; Furihata, Y.; Sakamoto, A.; Itoh, K.; Okumura, Y.; Tsuchida, T.; Kakehi, A.; Baba, T. Asymmetric cycloaddition reactions of diazoesters with 2-alkenoic acid derivatives catalyzed by binaphthyldiimine-Ni(II) complexes. J. Org. Chem. 2011, 76, 7377-7387.

29. Alex, K.; Tillack, A.; Schwarz, N.; Beller, M. Zinc-catalyzed synthesis of pyrazolines and pyrazoles via hydrohydrazination. Org. Lett. 2008, 10, 2377-2379.

30. Cui, S.-L.; Wang, J.; Wang, Y.-G. Facile access to pyrazolines via domino reaction of the Huisgen zwitterions with aziridines. Org. Lett. 2008, 10, 13-16.

31. Wang, Y.; Hu, W.J.; Song, W.; Lim, R.K.V.; Lin, Q. Discovery of long-wavelength photoactivatable diaryltetrazoles for bioorthogonal 1,3-dipolar cycloaddition reactions. Org. Lett. 2008, 10, 3725-3728. 
32. Li, F.; Xie, Z.F.; Liu, F.M. Syntheses and fluorescent property of 5-(2-Phenyl-1,2,3-triazoly)-3aryl pyrazoline derivatives. Chem. J. Chin. U 2006, 26, 1058-1061 (in Chinese).

33. Fischer, E.; Knovenagel, O. Ueber die verbindungen des phenylhydrazins mit acroleïn, mesityloxyd und allylbromid. Ann. Chem. 1887, 239, 194-206.

34. Azarifar, D.; Maleki, B. Silica-supported synthesis of some 1,3,5-trisubstituted 2-pyrazolines under solvent-free and microwave irradiation conditions. J. Heterocycl. Chem. 2005, 42, 157-159.

35. Levai, A. Synthesis of heterocyclic compounds by the reactions of exocyclic $\alpha, \beta$-unsaturated ketones. J. Heterocycl. Chem. 2004, 41, 299-310.

36. Levai, A. Synthesis of 2-pyrazolines by the reactions of $\alpha, \beta$-unsaturated aldehydes, ketones, and esters with diazoalkanes, nitrile imines, and hydrazines. J. Heterocycl. Chem. 2002, 39, 1-13.

37. Joshi, M.J.; Vekariya, P.B.; Dodiya, B.L.; Ghetiya, R.M.; Joshi, H.S. Synthesis and biological study of some new chalcones and oxopyrimidines containing imidazo[1,2-a]pyridine nucleus. J. Heterocycl. Chem. 2012, 49, 130-134.

38. Trilleras, J.; de la Torre, P.; Pacheco, D.J.; Quiroga, J.; Nogueras, M.; Cobo, J. Solvent-free microwave-assisted synthesis o substituted pyridines using $\mathrm{NH}_{4} \mathrm{OAc}$ as nitrogen source. Lett. Org. Chem. 2011, 8, 652-655.

39. Rostom, S.A.F.; Badr, M.H.; El Razik, H.A.A.; Ashour, H.M.A.; Abdel Wahab, A.E. Synthesis of some pyrazolines and pyrimidines derived from polymethoxy chalcones as anticancer and antimicrobial agents. Arch. Pharm. 2011, 344, 572-587.

40. Foroumadi1, A.; Emami, S.; Sorkhi, M.; Nakhjiri, S.; Nazarian, Z.; Heydari, S.; Ardestani, S.K.; Poorrajab, F.; Shafiee1, A. Chromene-based synthetic chalcones as potent antileishmanial agents: Synthesis and biological activity. Chem. Biol. Drug Des. 2010, 75, 590-596.

41. Sivakumar, P.M.; Ganesan, S.; Veluchamy, P.; Doble, M. Novel chalcones and 1,3,5-triphenyl-2pyrazoline derivatives as antibacterial agents. Chem. Biol. Drug Des. 2010, 76, 407-411.

42. Sreevidya, T.V.; Narayana, B.; Yathirajan, H.S. Synthesis and characterization of some chalcones and their cyclohexenone derivatives. Cent. Eur. J. Chem. 2010, 8, 174-181.

43. Voskienè, A.; Mickevičius, V. Cyclization of chalcones to isoxazole and pyrazole derivatives. Chem. Heterocycl. Compd. 2009, 45, 1485-1488.

44. Cella, R.; Stefani, H.A. Ultrasound in heterocycles chemistry. Tetrahedron 2009, 65, 2619-2641.

45. Li, J.-T.; Zhang, X.-H.; Lin, Z.-P. An improved synthesis of 1,3,5-triaryl-2-pyrazolines in acetic acid aqueous solution under ultrasound irradiation. Beilstein J. Org. Chem. 2007, 3, 1-4.

46. Li, J.-T.; Yang, W.-Z.; Wang, S.-X.; Li, S.-H.; Li, T.-S. Improved synthesis of chalcones under ultrasound irradiation. Ultrason. Sonochem. 2002, 9, 237-239.

47. Zare, L.; Mahmoodi, N.O.; Yahyazadeh, A.; Nikpassand, M. Ultrasound-promoted regio and chemoselective synthesis of pyridazinones and phthalazinones catalyzed by ionic liquid [bmim] $\mathrm{Br} / \mathrm{AlCl}_{3}$. Ultrason. Sonochem. 2012, 19, 740-744.

48. Saleh, T.S.; El-Rahman, N.M.A.; Elkateb, A.A.; Shaker, N.O.; Mahmoud, N.A.; Gabal, S.A. Ultrasound promoted synthesis of some novel fused pyrans. Ultrason. Sonochem. 2012, 19, 491-497.

49. Zhang, Z.-H.; Li, J.-J.; Li, T.-S. Ultrasound-assisted synthesis of pyrroles catalyzed by zirconium chloride under solvent-free conditions. Ultrason. Sonochem. 2012, 19, 264-269. 
50. Nagargoje, D.; Mandhane, P.; Shingote, S.; Badadhe, P.; Gill, C. Ultrasound assisted one pot synthesis of imidazole derivatives using diethyl bromophosphate as an oxidant. Ultrason. Sonochem. 2012, 19, 94-96.

51. Dabiri, M.; Noroozi Tisseh, Z.; Bahramnejad, M.; Bazgir, A. Sonochemical multi-component synthesis of spirooxindoles. Ultrason. Sonochem. 2011, 18, 1153-1159.

52. Trilleras, J.; Insuasty, B.; Abonía, R.; Nogueras, M.; Cobo, J. Síntesis y estudio de la actividad antitumoral y antituberculosa de análogos heterocíclicos enónicos derivados del pirazol. Revista Ciencias 2008, 12, 123-140.

53. Trilleras, J.; Quiroga, J.; Cobo, J.; Low, J.N.; Glidewelld, C. Hydrogen-bonded chains in 3-(5chloro-3-methyl-1-phenyl-1H-pyrazol-4-yl)-1-(4-methoxyphenyl)-propenone and 3-(5-chloro-3methyl-1-phenyl-1H-pyrazol-4-yl)-1-(3,4,5-trimethoxyphenyl)propenone. Acta Cryst. 2005, C61, 414-416.

54. Trilleras, J.; Quiroga, J.; Cobo, J.; Low, J.N.; Glidewelld, C. 5-Chloro-3-methyl-1-phenyl-1Hpyrazole-4-carbaldehyde: Sheets built from $\mathrm{C}-\mathrm{H}---\mathrm{O}$ and $\mathrm{C}-\mathrm{H}---\pi($ arene) hydrogen bonds. Acta Cryst. 2005, E61, 1055-1057.

55. Xie, Z.; Moa, Z.; Liu, G.; Liu, F. Crystal structure of 5-pyrazol-4,5-dihydropyrazoles derivatives. J. Heterocycl. Chem. 2008, 45, 1485-1488.

56. Ziarati, A.; Safaei-Ghomi, J.; Rohani, S. Sonochemically synthesis of pyrazolones using reusable catalyst $\mathrm{CuI}$ nanoparticles that was prepared by sonication. Ultrason. Sonochem. 2013, 20, 1069-1075.

57. Shekouhy, M.; Hasaninejad, A. Ultrasound-promoted catalyst-free one-pot four component synthesis of $2 \mathrm{H}$-indazolo[2,1-b]phthalazine-triones in neutral ionic liquid 1-butyl-3methylimidazolium bromide. Ultrason. Sonochem. 2012, 19, 307-313.

58. Mason, T.J. Sonochemistry sonoprocessing: The link the trends and (pobably) the future. Ultrason. Sonochem. 2003, 10, 175-179.

(C) 2013 by the authors; licensee MDPI, Basel, Switzerland. This article is an open access article distributed under the terms and conditions of the Creative Commons Attribution license (http://creativecommons.org/licenses/by/3.0/). 